Lastly, how did environmental changes, human migrations and cultural and genetic adaptations interplay in northeastern Siberia and the far northern Americas? The two latest studies will help us to get our bearings as we work to understand the ancient humans who lived around the Bering Strait..

Anne C. Stone is in the School of Human Evolution and Social Change, Arizona State
University, Tempe, Arizona 85287, USA. e-mail:acstone@asu.edu

1. Nogués-Bravo, D., Rodríguez, J., Hortal, J., Batra, P. \& Araújo, M. B. PLoS Biol. 6, e79 (2008).

2. Pitulko, V., Pavlova, E. \& Nikolskiy, P. Quat. Sci. Rev. 165, 127-148 (2017)

3. Hoffecker, J. F., Elias, S. A., O'Rourke, D. H., Scott, G. R. \& Bigelow, N. H. Evol. Anthropol. 25, 64-78 (2016)

4. Sikora, M. et al. Nature 570, 182-188 (2019).

5. Flegontov, P. et al. Nature 570, 236-240 (2019).

6. Pitulko, V. V. et al. Science 351, 260-263 (2016).
7. Hu, A. et al. Nature Geosci. 3, 118-121 (2010).

8. Pitulko, V. V. \& Nikolskiy, P. A. World Archaeol. 44, 21-42 (2012).

9. Fagundes, N. J. R. et al. Am. J. Hum. Genet. 82, 583-592 (2008).

10.Goebel, T., Waters, M. R. \& O'Rourke, D. H. Science 319, 1497-1502 (2008).

11. Raghavan, M. et al. Science 345, 1255832 (2014).

12. Tamm, E. et al. PloS ONE 2, e829 (2007).

13. Raghavan, M. et al. Science 349, aab3884 (2015).

14.Skoglund, P. et al. Nature 525, 104-108 (2015).

This article was published online on 5 June 2019.

\section{Catalytic machinery of enzymes expanded}

Only a few types of natural amino-acid residue are used directly by enzymes to catalyse reactions. The incorporation of an unnatural residue into an enzyme shows how the catalytic repertoire of enzymes can be enlarged. SEE LETTER P. 219

\section{ADAM NELSON}

$\mathrm{E}$ nzymes are exceptionally powerful catalysts that recognize molecular substrates and process them in active sites. They are generally built from just 20 types of amino acid, and their catalytic machinery is typically assembled from chemical groups in the amino-acid side chains, often with extra bound metal ions or cofactors. This raises the question of whether the catalytic repertoire of enzymes could be expanded by using an extended 'alphabet' of amino acids that offers a wider range of side chains for catalysis. On page 219, Burke et al. ${ }^{1}$ report the construction of an enzyme that uses an unnatural catalytic chemical group, and show that the enzyme's catalytic properties can be greatly improved using an approach called directed evolution.
The amino-acid side chains found in enzymes contain at most one chemical group, and are crucial for molecular recognition. But fewer than half of these side chains contain groups that can act as acids, bases or nucleophiles (electron-pair donors) in enzyme catalytic cycles. None of the side chains can act as electrophiles (electron-pair acceptors), which could also be useful for catalysis. The introduction of unnatural amino-acid residues that bear potentially catalytic side chains could therefore open up a wide range of new enzymatic reactions.

Conventional catalysts are a fertile source of inspiration for chemical groups that would expand the catalytic repertoire of enzymes: both small-molecule organic catalysts (organocatalysts) and transition-metal catalysts can activate substrate molecules in ways

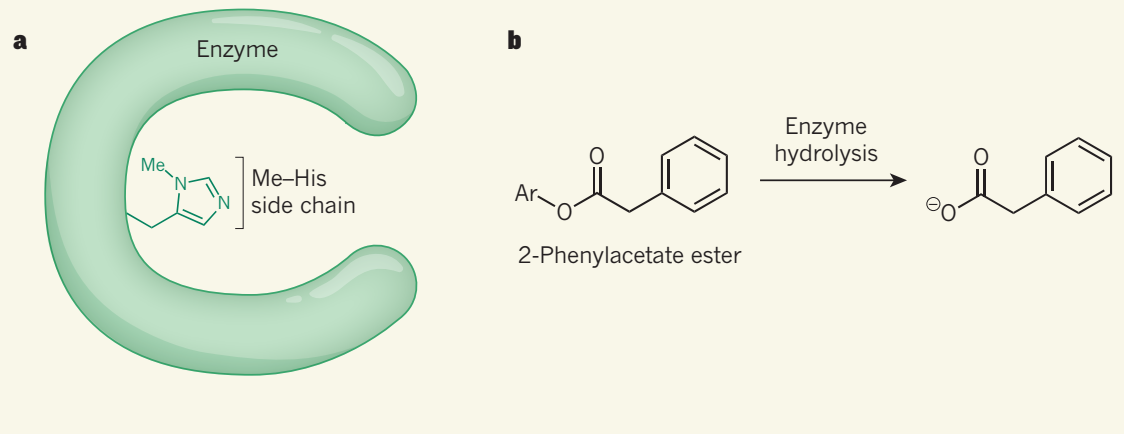

Figure 1 | An unnatural amino-acid residue remodels enzyme activity. a, Burke et al. ${ }^{1}$ have replaced a histidine amino-acid residue in an enzyme's active site with an unnatural residue $-N_{\delta}$-methylhistidine (Me-His), an analogue of histidine in which a methyl group (Me) is attached to one of the nitrogen atoms in the side chain. $\mathbf{b}$, The authors optimized the resulting enzyme using a method called directed evolution, thereby producing an enzyme that catalyses the hydrolysis of compounds called 2 -phenylacetate esters. This reaction is very different from the one that the starting enzyme had been designed to catalyse. Ar is a group that generates a fluorescent by-product on hydrolysis. that enable a variety of reactions that are useful for organic synthesis. To enable enzymes to access this exciting reactivity, methods are required for the efficient site-specific incorporation of amino acids that bear new chemical groups. Methods for the directed evolution of the resulting modified enzymes are also required to optimize catalysis in active sites.

Artificial enzymes have previously been constructed by attaching transition-metal catalysts to a small molecule known as biotin, which in turn binds non-covalently with extremely high affinity to the protein streptavidin, thus anchoring the catalyst in a protein framework $^{2,3}$. Metal catalysts have also been covalently attached to the side chains of unnatural amino-acid residues that have been incorporated into proteins using modified biological protein-synthesis machinery ${ }^{4}$. With both of these strategies, directed evolution was used to greatly improve the catalytic efficiency and turnover (the average number of reactions catalysed by each enzyme) of the initially produced artificial enzymes, and, in some cases, to increase the selectivity of the enzyme for a particular mirrorimage isomer of the product (enantioselectivity). Artificial enzymes have thus been produced that catalyse reactions not found in nature, including silicon-carbon bondforming reactions ${ }^{4}$, and carbon-carbon bond-forming reactions known as cyclopropanations ${ }^{4}$ and ring-closing metathesis reactions ${ }^{2}$.

Burke et al. took a different approach. They started from an enzyme ${ }^{5}$ (BH32) that had been computationally designed to catalyse a particular type of carbon-carbon bond-forming reaction, but which also weakly catalyses an unrelated transformation: the hydrolysis of compounds known as 2-phenylacetate esters (Fig. 1). The authors therefore decided to remodel the enzyme to make it an effective catalyst for these hydrolyses.

The researchers determined that a histidine amino-acid residue (His 23) in $\mathrm{BH} 32$ forms an intermediate called an acyl-enzyme compound during the catalytic cycle. This intermediate is then hydrolysed to yield the product of the enzymatic reaction. However, the catalytic turnover was poor because the hydrolysis of the acyl-enzyme intermediate was slow.

To address this issue, Burke and colleagues replaced His23 with a genetically encodable, unnatural amino acid called $N_{\delta}$-methylhistidine (Me-His; Fig. 1). Me-His 
is an analogue of histidine in which a methyl group is attached to one of the nitrogen atoms in the side chain. The authors observed that catalytic turnover for the modified enzyme (OE1) was higher than for $\mathrm{BH} 32$, an effect that they ascribed to more rapid hydrolysis of the acyl-enzyme intermediate.

Burke et al. then used directed evolution to optimize the function of Me-His in the enzyme's active site. A wide range of strategies was used to introduce mutations, ultimately resulting in the discovery of a variant, OE1.3, that had improved catalytic efficiency. This variant differed from OE1 by having six mutations, in which one amino-acid residue has been replaced by another. The authors found that OE1.3 hydrolyses a range of analogues of 2-phenylacetate esters in which only hydrogen atoms are attached to the carbon atom adjacent to the carbonyl $(\mathrm{C}=\mathrm{O})$ group in the molecules. However, analogues in which a methyl group is attached next to the carbonyl group were poor substrates. The authors therefore carried out further directed evolution to generate OE1.4, an enzyme that has improved catalytic activity with this class of substrate, and which predominantly hydrolyses one of the two mirror-image isomers of each substrate.

The Me-His residue in the modified enzymes acts as a nucleophilic catalyst that is broadly analogous to the nucleophilic residues found in serine hydrolase and cysteine hydrolase enzymes. But how might organocatalysis ${ }^{6}$ in general inspire the discovery of enzymes that are more distant from those found in nature? Organocatalysts speed up many different reactions using just a few generic mechanisms (activation modes), but the catalysis is often inefficient, requiring rather high catalyst loadings (typically 5-20 mole \%) ${ }^{6}$. Some of these activation modes are also widely used by enzymes; for example, enamine catalysis is used by class I aldolases ${ }^{7}$. But other activation modes are less widely used enzymatically, despite the fact that they can enable many potentially useful synthetic reactions.

Organocatalysts have been introduced into proteins in various ways, for example by using an attached biotin group as an anchor that binds to streptavidin ${ }^{8}$, or by chemically modifying genetically encoded unnatural amino-acid residues ${ }^{9}$. However, to realize the full power of an expanded range of catalytic chemical groups, substantial optimization is likely to be needed to generate catalytically efficient active sites. Burke et al. have shown that directed evolution can improve enzymes that contain an unnatural organocatalytic group. Their approach might also provide a route to efficient enzymes that use activation modes not found in nature, and which are much more efficient than organocatalysts themselves.

Adam Nelson is at the School of Chemistry and at the Astbury Centre for Structural Molecular Biology, University of Leeds,

Leeds LS2 9JT, UK.

e-mail:a.s.nelson@leeds.ac.uk

1. Burke, A. J. et al. Nature 570, 219-223 (2019).

2. Jeschek, M. et al. Nature 537, 661-665 (2016).

3. Heinisch, T. et al. Chem. Sci. 9, 5383-5388 (2018).

4. Yang, H. et al. Nature Chem. 10, 318-324 (2018).

5. Bjelic, S. et al. ACS Chem. Biol. 8, 749-757 (2013).

6. MacMillan, D. W. C. Nature 455, 304-308 (2008).

7. Windle, C., Müller, M., Nelson, A. \& Berry, A. Curr. Opin. Chem. Biol. 19, 25-33 (2014).

8. Nödling, A. R. et al. Angew. Chem. Int. Edn 57 12478-12482 (2018)

9. Drienovská, I., Mayer, C., Dulson, C. \& Roelfes, G. Nature Chem. 10, 946-952 (2018).

This article was published online on 27 May 2019.

\section{Bacterial dormancy curbs phage epidemics}

One type of CRISPR-Cas bacterial-defence system destroys phage and bacterial RNA, which leads to bacterial dormancy. Dormancy is found to limit viral spread, and also protects against unrelated viruses and viral mutants. See LETTER P.241

\section{SIMON A. JACKSON \& PETER C. FINERAN}

A $\mathrm{n}$ unseen war is being waged right under our noses, between microorganisms and their viral invaders. To fight the viruses called bacteriophages (phages) that target them, bacteria have evolved a diverse armoury of defences, which includes a range of protective immune systems called CRISPR-Cas.

On page 241, Meeske et al. ${ }^{1}$ reveal a curious twist in our understanding of the incredible variety of CRISPR-Cas defences, by demonstrating how a type of the CRISPR-Cas system that targets phage RNA protects bacteria from infection by DNA phages. The authors report that this CRISPR-Cas system responds to DNA phages by unleashing the destruction of both viral and bacterial RNA, which then causes infected bacterial cells to enter a state of dormancy that shuts down the cellular processes needed for viral replication. Meeske and colleagues reveal that this self-induced bacterial dormancy helps to suppress viral replication and viral outbreaks, including those caused by viral mutants that can escape other types of CRISPR-Cas defences, or viruses unrelated to the one that triggered dormancy. This defence response, in which the shutdown of an infected bacterial cell might benefit neighbouring bacteria, has interesting parallels with other types of defence system, such as bacterial abortive-infection

"These dormant
cells can act
as sacrificial
'decoy' cells."
systems or cell death in plants and animals that is induced by the innate branch of the immune system.

CRISPR-Cas systems are classified into six types termed I to VI. Most such systems capture and store short sequences of viral DNA as genetic 'memories' of phage invasion. These stored sequences are used to generate RNA guides that enable Cas enzymes to target and degrade viral DNA or RNA. Type VI systems are intriguing because they are the only ones that destroy viral RNA
(Fig. 1) rather than $\mathrm{DNA}^{2-5}$, yet most phages have DNA rather than RNA genomes.

Type VI CRISPR-Cas systems, which use a Cas enzyme called Cas13, have previously been shown ${ }^{2,3}$ to respond to infection by RNA viruses by activating a form of indiscriminate (low sequence specificity) RNA-degrading activity by Cas13. In addition, when bacteria were engineered so that Cas 13 targeted a messenger RNA encoded by a circular DNA sequence called a plasmid, bacterial growth was impaired ${ }^{2,6}$. This suggested that, in the absence of phage infection, Cas13 activation, and its indiscriminate RNA destruction, led to bacterial-cell dormancy. But what role dormancy has, if any, in the antiviral defence processes remained an unanswered question.

To address this issue, Meeske et al. studied a type VI defence system using the bacterium Listeria ivanovii and the DNA phage $\phi R R 4$. The authors engineered the type VI system to guide Cas13 to target different $\phi R R 4$ viral sequences and then analysed how effectively this system provided antiviral defence. Cas 13 did indeed provide defence when targeted to viral mRNAs and, surprisingly, protection was achieved regardless of whether or not the targeted viral mRNAs corresponded to genes that are essential for viral replication, or whether the genes were expressed early or late during viral infection. There was also extensive bacterial RNA degradation in the infected cells, which caused infected bacteria to enter a dormant state in which the bacterial cells were alive but could not replicate.

This form of Cas13-induced dormancy has considerable parallels with another class of phage-defence system called 